\title{
DESIGN AND EVALUATION OF NEXTGEN AIRCRAFT SEPARATION ASSURANCE CONCEPTS
}

\author{
Nhut Ho ${ }^{1}$, Walter Johnson ${ }^{2}$, Vladimir Arutyunov ${ }^{1}$, John-Luke Laue ${ }^{1}$, and Ian Wilmoth ${ }^{1}$ \\ ${ }^{l}$ California State University Northridge, Northridge, $C A$ \\ ${ }^{2}$ NASA Ames Research Center, Moffett Field, CA
}

\begin{abstract}
To support the development and evaluation of future function allocation concepts for separation assurance systems for the Next Generation Air Transportation System, this paper presents the design and human-in-the-loop evaluation of three feasible function allocation concepts that allocate primary aircraft separation assurance responsibilities and workload to: 1) pilots; 2) air traffic controllers (ATC); and 3) automation. The design of these concepts also included rules of the road, separation assurance burdens for aircraft of different equipage levels, and utilization of advanced weather displays paired with advanced conflict detection and resolution automation. Results of the human-in-theloop simulation show that: a) all the concepts are robust with respect to weather perturbation; b) concept 1 (pilots) had highest throughput, closest to assigned spacing, and fewest violations of speed and altitude restrictions; c) the energy of the aircraft during the descent phase was better managed in concepts 1 and 2 (pilots and ATC) than in concept 3 (automation), in which the situation awareness of pilots and controllers was lowest, and workload of pilots was highest. The paper also discusses further development of these concepts and their augmentation and integration with future air traffic management tools and systems that are being considered for NextGen.
\end{abstract}

\section{Introduction}

The current National Airspace System (NAS) is under stress and air traffic density is expected to increase twofold to threefold by 2025. If not addressed, this trend will lead to billions of dollars of lost economic activity, increased safety risks, and decreased system reliability and stability. Managed by the Joint Planning and Development Office (JPDO), the Next Generation Air Transportation System (NextGen) is an initiative that addresses these issues through a comprehensive and ongoing transformation of the NAS with continuous deployment of improvements and updates implemented in stages between 2012 and 2025. NextGen's goals are to increase the NAS' efficiency, capacity, and security, while maintaining or improving safety, and to reduce the environmental impact of aviation $[1,2]$.

To achieve these goals, new system operating principles and capabilities are being conceived and developed under NextGen. An important area that has received considerable research interest is the development of separation assurance concepts that involve significant changes to the roles and responsibilities of the air traffic controllers (ATCs), pilots, and ground-based and airborne automation systems. The concepts vary by the extent to which the separation assurance function is distributed among pilots, controllers, and automation. One of the main issues that these concepts address is the significant increase in the ATC workload that comes with increased air traffic densities. Automation, in particular, is a primary means by which designers are seeking to address this workload issue and thereby safely increase the allowable air traffic densities. Methods by which automation can be brought to bear on this problem may be defined with respect to the relative degree of human involvement in generating resolutions for conflicts. A number of current research efforts aim to develop automated systems which detect projected losses of separation (conflicts) and then generate conflict resolutions (trajectory modifications), thereby, substantially augmenting and/or replacing functions now performed by ATCs [3-6]. For instance, the groundbased automation tool developed under the Advanced Airspace Concept (AAC) by researchers at NASA Ames $[7,8]$ is designed to detect conflicts and then to generate and transmit conflict-free routes (via datacom) to appropriately equipped aircraft for execution. In this concept, separation assurance could be managed jointly by the ground based automation system and pilots, while controllers are 
mainly responsible for strategic management of air traffic flow and separation of unequipped aircraft. An alternative to the above approach has also been proposed in which the controller delegates the separation responsibility to the pilot. The consensus view in the NextGen community is that this approach would require advance airborne automation in the form of a cockpit situation display (CSD) and conflict detection and resolution (CD\&R) tools for separation management [9-17].

While the benefits of automation have been shown, the exact roles and responsibilities of pilots, controllers, and automation surrounding these NextGen concepts are still in development. In 2009 a human-in-the-loop simulation was conducted to help support the development and evaluation of allocating separation assurance responsibilities and workload to these three entities. The study presented flights through and around weather during the cruise phase of flight, and for which the differing allocations were examined. Previous papers have reported subjective and performance data for the cruise phase in this study [18-19]. However, each of the flights in this study culminated with a final weather free arrival phase down to the meter fix during which ATCs were always responsible for separation management, but there has been only a limited examination of how the cruise phase functional allocation affected performance associated with the arrival phase. This paper presents a more detailed analysis of this aspect of the study. Section II describes the design of the three concepts using a human-centered approach, Section III describes the experimental design and methods of the human-in-the loop simulation used to evaluate these concepts, while Sections IV and V discuss this study's results, their implications for further development of these concepts, and their augmentation and integration with future air traffic management tools and systems that are being considered for NextGen.

\section{Design of Concepts}

Three function allocation concepts were conceived and designed with a human-centered approach that distributes separation assurance responsibility among pilots, controllers, and air/ground automation. Specific divisions of functions were chosen to vary operator workload and situation awareness, and to examine system performance across concepts. A set of common assumptions regarding aircraft equipage, data communication technologies, airborne and groundbased automated decision support tools, and rules of the road for conflict management were developed. These assumptions include:

1. There are two groups of aircraft operating in the airspace: a) $50 \%$ of all aircraft are trajectory flight rule (TFR) aircraft, and have a cockpit situation display (CSD) on board integrated with a route assessment (trajectory replanning) tool (RAT) and a 3D-weather display [20] plus, in some cases, CD\&R tools. Using the RAT, the pilot can manually make changes to the trajectory; and b) $50 \%$ of all aircraft are instrument flight rule aircraft (IFR) managed by ATC and do not have CD\&R tools. In this study, all experimental pilots flew TFR aircraft.

2. All aircraft have the capability to communicate and exchange information with ATC through Controller Pilot Data Link Communications (CPDLC) and Automatic Dependent Surveillance Broadcasting (ADS-B).

3. Pilots of both TFR and IFR aircraft are responsible for interval management operations [15], using flight deck automation [11] to space $105 \mathrm{sec}$ behind an assigned lead by the final approach fix.

4. The ground and (when present) airborne auto-resolver systems use the NASA Advanced Airspace Concept (AAC) [7, 8] algorithm for detection and resolution of conflicts between 4 and 12 minutes to predict loss of separation (LOS), and use the Tactical Separation Assisted Flight Environment (TSAFE) algorithm for avoidance of conflicts less than 4 minutes to LOS. The autoresolver tools on the ground and in the flight deck do not take weather into account; thus, pilots must ensure all resolutions are weather free.

5. To resolve a conflict, and if equipped, the TFR pilot can use either the airborne auto-resolver on board to generate a conflict-free resolution and check for weather-free, or the RAT to do the same tasks. Similarly, the controller can use either the groundbased auto-resolver to generate resolutions and check for weather-free, or the manual trial planning tool to do the same tasks.

6. Rules of the road: When in conflict with IFR aircraft, operators or automation must resolve the conflict by changing the trajectory of the TFR aircraft. For conflicts that are 4 minutes or less to 
LOS, TSAFE provides conflict-free resolutions to the controller for both TFR and IFR aircraft.

With these assumptions providing a baseline for the operation, three function allocation concepts were developed to vary the responsibility for conflict resolution as well as the amount of conflicts that pilots, controllers, and automation must manage. The three concepts are summarized below:

\section{Concept 1: Pilot Primary, Controller Secondary}

TFR pilots are equipped with a conflict probe tool that detects conflicts and alerts the operator, and a conflict resolution tool with which the operator can either request algorithmically-generated conflict resolutions or create their own conflict resolutions. TFR pilots are responsible for generating reroutes to avoid weather and for identifying and resolving TFRTFR and TFR-IFR conflicts with ownship. IFR pilots are responsible only for verbally requesting reroutes around weather. Controllers are equipped with equivalent ground-based tools and responsible for IFR-IFR conflicts. Controllers managed all IFR aircraft. When avoiding traffic and weather, pilots self-modify and execute their routes and broadcast their route modifications via ADS-B, thereby updating other TFR aircraft and the air traffic control system. Pilots monitor voice frequencies but will only receive clearances from the controller if their spacing becomes discontinuous and responsibility is delegated to the controller to adjust. Through these allocations, pilots are responsible for resolving $75 \%$ of the total conflicts, ATC is responsible for $25 \%$ of the total conflicts, and the Auto-resolver agent is not responsible for resolving any conflicts.

\section{Concept 2: Controller Primary, Automation Secondary}

TFR pilots are equipped with a conflict probe tool and a conflict resolution tool, as they are in Concept 1, but they are not responsible for resolving any conflicts. Instead, they have the capability of generating conflict and weather free reroutes, and datalinking them to the controller, but the controller must approve/disapprove or offer alternatives to all such reroutes. Both IFR and TFR pilots are responsible for requesting reroutes around weather. Controllers are equipped with equivalent groundbased tools and are responsible for generating and/or approving TFR-IFR and IFR-IFR conflict resolutions. For TFR-IFR conflicts, the controller modifies the route of the IFR aircraft unless an acceptable TFR resolution is datalinked to the controller. An autoresolver agent, which acts independently when responsibility is delegated to it and datalinks route modifications directly to operators, is responsible for TFR-TFR conflicts, but does not take weather into account. While the controller is not in the decision-making loop for initial resolution of TFR-TFR conflicts, TFR pilots can review route modifications made by the autoresolver agent before executing them, and return them to the controller if not weather free. Through these allocations, pilots are not responsible for resolving any conflicts, ATC is responsible for resolving $75 \%$ of the total conflicts, and the autoresolver agent is responsible for resolving $25 \%$ of the total conflicts.

\section{Concept 3: Automation Primary, Controller Secondary}

TFR pilots are equipped with a CSD with the RAT flight path replanning tool, but without CD\&R tools. As in Concept 2, pilots are responsible for requesting and obtaining weather free re-routes, but are not responsible for resolving any conflicts. The autoresolver agent is responsible for resolving TFRTFR and TFR-IFR conflicts, but as in Concept 2 it does not take weather into account. For TFR-IFR conflicts, the autoresolver agent preferentially modifies the route of the TFR aircraft. Controllers are equipped with a conflict probe tool and a conflict resolution tool and are responsible for IFR-IFR conflicts. TFR pilots can review route modifications datalinked to them by the autoresolver agent before executing them. TFR pilots use the CSD to generate and datalink reroute requests for weather avoidance to the Autoresolver but, if the reroute has conflicts, it will be automatically transferred to, and subsequently handled by, the controller. Through these allocations, pilots are not responsible for resolving any conflicts, ATC is responsible for resolving $25 \%$ of the total conflicts, and the auto-resolver agent is responsible for resolving $75 \%$ of the total conflicts.

In all three concepts once the aircraft reached the top of descent, the controller became solely responsible for separation, while the pilots remained responsible for achieving a 105 second in trail separation by the final approach fix. The separation responsibility and equipage assignment are summarized in Table 1 below. 
Table 1. Equipages and responsibilities of pilots, controllers, and automation in each concept

\begin{tabular}{|c|l|l|}
\hline $\begin{array}{c}\text { Flight } \\
\text { Phase }\end{array}$ & Cruise & Descent/Arrival \\
\hline $\begin{array}{c}\text { Concept } \\
\mathbf{1}\end{array}$ & $\begin{array}{l}\text { Pilots: Equipped with CD\&R and interval } \\
\text { management automation, responsible for TFR- } \\
\text { TFR and TFR-IFR conflicts and spacing. } \\
\text { Controllers: CD\&R equipped and responsible for } \\
\text { IFR-IFR conflicts } \\
\text { Auto-resolver Agent: Responsible for no conflicts. }\end{array}$ & $\begin{array}{l}\text { Pilots: No CD\&R, responsible for no } \\
\text { management automation, responsible for } \\
\text { spacing goals. } \\
\text { Controller: CD\&R equipped, responsible for } \\
\text { all conflicts }\end{array}$ \\
\hline $\begin{array}{c}\text { Concept } \\
\mathbf{2}\end{array}$ & $\begin{array}{l}\text { Pilots: Equipped with CD\&R and interval } \\
\text { management automation, responsible for spacing } \\
\text { but not for conflicts. } \\
\text { Controllers: CD\&R equipped and responsible for } \\
\text { TFR-IFR and IFR-IFR conflicts } \\
\text { Auto-resolver Agent: Responsible for TFR-TFR } \\
\text { conflicts. }\end{array}$ & $\begin{array}{l}\text { Pilots: No CD\&R, not responsible for any } \\
\text { automation, responsible for spacing goals. } \\
\text { Controller: CD\&R equipped, responsible for }\end{array}$ \\
\hline $\begin{array}{c}\text { all conflicts } \\
\mathbf{3}\end{array}$ & $\begin{array}{l}\text { Pilots: Not CD\&R equipped or responsible for any } \\
\text { conflicts. Equipped with interval management } \\
\text { automation, responsible for spacing goals. } \\
\text { Controllers: CD\&R equipped and responsible for } \\
\text { IFR-IFR conflicts. } \\
\text { Auto-resolver Agent: Responsible for TFR-TFR } \\
\text { and TFR-IFR conflicts. }\end{array}$ & $\begin{array}{l}\text { Pilots: No CD\&R, not responsible for any } \\
\text { conflicts. Equipped with interval management } \\
\text { automation, responsible for spacing goals. } \\
\text { Controller: CD\&R equipped, responsible for } \\
\text { all conflicts }\end{array}$ \\
\hline
\end{tabular}

\section{Experiment Design}

To evaluate the impact of functional allocation concept used during cruise on system performance during arrival, and on the workload and situation awareness of pilots and controllers during arrival, a human-in-the-loop (HITL) simulation was developed. The simulation airspace assumes a 3-time current day traffic density operating in both the enroute cruise and arrival flight phases under the three separation assurance functional allocation concepts described above.

\section{III.A. Scenario and Testing Design}

Traffic scenarios were created with aircraft being assigned an interval management clearance (designating lead aircraft and $105 \mathrm{sec}$ in trail) prior to encountering convective weather in the cruise phase of flight. They were then required to avoid the weather, and perform a continuous descent approach (CDA) arrival from the top of descent (TOD) into Louisville Standiford Field Airport (SDF) using the CBSKT-1 arrival shown top-down in Figure 1.

The simulated airspace, modeled after Kansas City Air Route Traffic Control Center (ZKC) and Indianapolis Air Route Traffic Control Center (ZID), consists of two airspace "super-sectors" ZKC 90 and ZKC 91, which are larger than current-day airspace. Super-sector ZKC90 was created by geographically combining existing ZKC sectors 90 and 14, while "Super-sector" ZID 91 was created by combining the existing ZID sectors 91, 81 and 17. This airspace was then populated with additional aircraft to create a 3-time current day traffic density. The traffic flow, modeled after real traffic streams, encountered enroute weather cells west of ZKC 90, and then reached the TOD before merging and performing the 


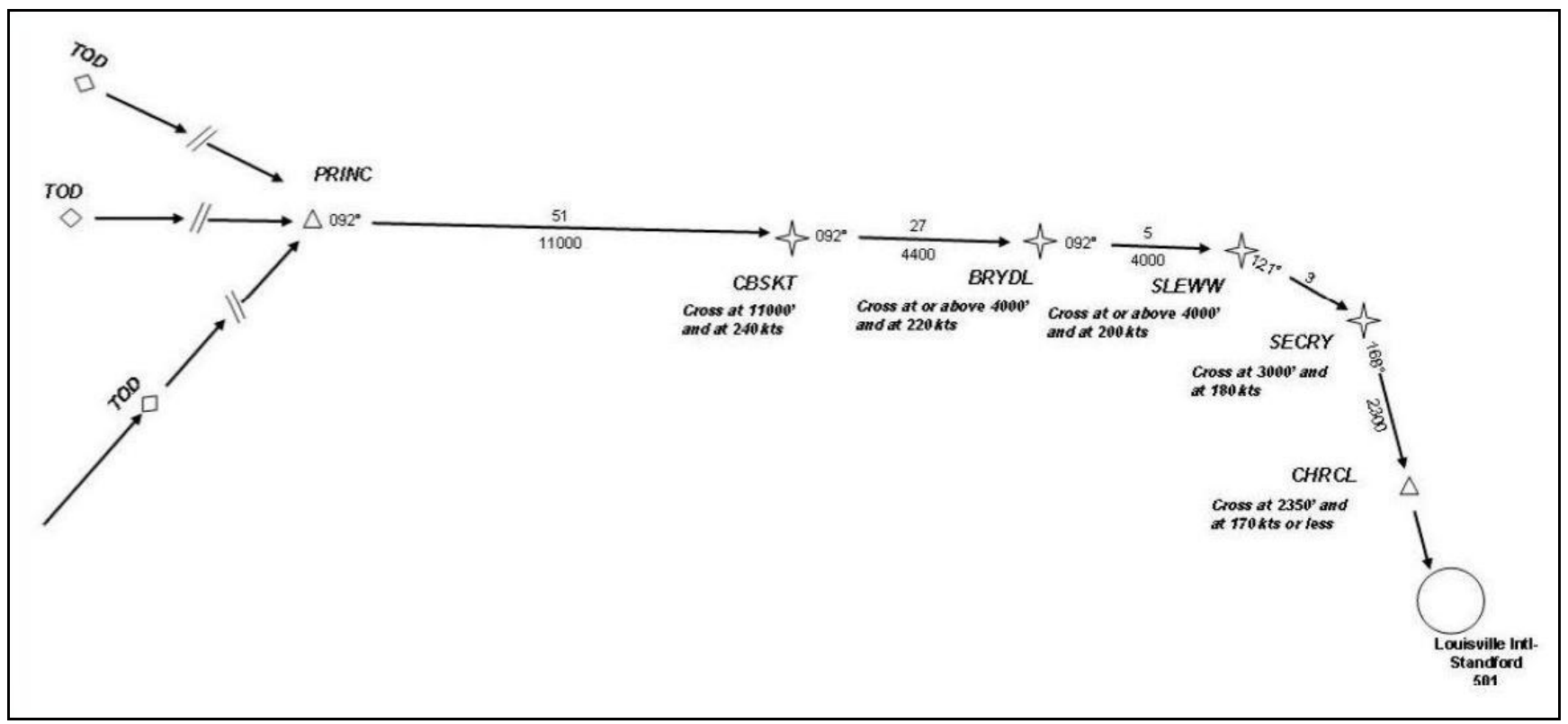

Figure 1. CBSKT-1 Arrival

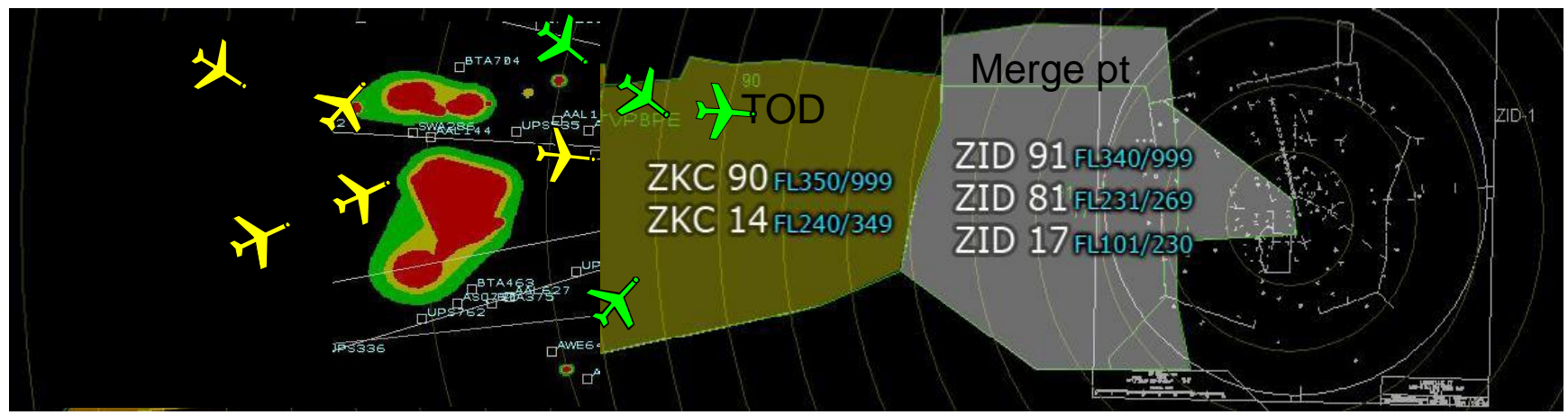

Figure 2. Airspace and sector layout

\section{III.B. Independent Variables and Analysis Metrics}

A 3 (Concept: 1 - Pilot Primary, 2 - Controller Primary, 3 - Automation Primary) x 2 (Weather Complexity: Low, High) x 2 (Cockpit Weather Display: airborne radar, 3D NexRad) fully withinsubjects factorial design was used. Specific numbers of TFR and IFR aircraft were designed into the scenarios to generate the following divisions of responsible conflicts count: $75 \%$ of conflicts to Pilots and $25 \%$ to Controllers for Concept $1 ; 75 \%$ of conflicts to Controllers and $25 \%$ to Automation for Concept 2; and $75 \%$ of conflicts to Automation and $25 \%$ to Controllers for Concept 3. Low weather density had fewer and sparser weather cells, while high weather density had greater and denser weather cells. However, in the analysis of this paper, neither the Weather Complexity nor Cockpit Weather Display manipulations are examined, and will not be further mentioned. Twelve trials were conducted, one for each unique set of conditions. The CSD provided pilots with $3 \mathrm{D}$ and $2 \mathrm{D}$ spatial orientations of traffic information.

Five types of data were collected to help quantify the effects of the function allocation during the arrival portion of the simulation: situation awareness data, aircraft trajectory data, voice data, workload data, and subjective ratings. Based on these data, a number of metrics were developed to quantify system performance and human factors performance. System performance, defined in terms of the system's stability, efficiency, and compliance 
with safety, was measured by five key metrics: 1) throughput at the final approach fix; 2) aircraft kinetic energy; 3) number of violation of speed and altitude constraints; 4) Spacing interval variation at the merge point and at the final approach fix; and 5) variation in speed and altitude along the descent. The human factors performance was primarily measured by the workload and situation awareness ratings.

\section{III.C. Participants}

Eight ATP pilots and two air traffic controllers participated each week of the two-week experiment. However, only data collected during the second week of this experiment is reported in this paper, due to equipment failure and malfunction occurred during the first week of the experiment. The pilots' flight time experience is summarized in Table 2 below [18]. Five of the eight second-week pilots were Captains and three were First Officers. Three pilots had previous experience flying CDAs but none of them had experience with merging and spacing operations. The two second-week controllers were retired, radar certified controllers, with 25 and 34 years of civilian air traffic control experience.

Table 2. Pilot flight hours

\begin{tabular}{|r|r|r|r|}
\hline $\begin{array}{l}\text { Total hours flown } \\
\text { as a line-pilot }\end{array}$ & $\mathbf{N}$ & $\begin{array}{l}\text { Total hours flown in } \\
\text { "glass" cockpit }\end{array}$ & $\mathbf{N}$ \\
\hline $1-1000$ & 1 & $1-1000$ & 4 \\
\hline $1001-3000$ & 0 & $1001-3000$ & 1 \\
\hline $3001-5000$ & 4 & $3001-5000$ & 3 \\
\hline$>5000$ & 3 & $>5000$ & 0 \\
\hline
\end{tabular}

\section{III.D. Simulation Equipment}

The HITL simulation used PC desktop-based single pilot and controller stations equipped with the Multi Aircraft Control System (MACS) and the Cockpit Situation Display (CSD) simulation software applications developed by NASA Ames Research Center's Airspace Operations Laboratory (AOL) and Flight Deck Display Research Laboratory (FDDRL) respectively. MACS and CSD interfaces, shown in Figures 3 and 4, are connected and supported by a number of supplementary communication and networking applications. The MACS controller display is an emulation of current day controller stations with a ground-based automated conflict probe tool that can automatically detect and alert

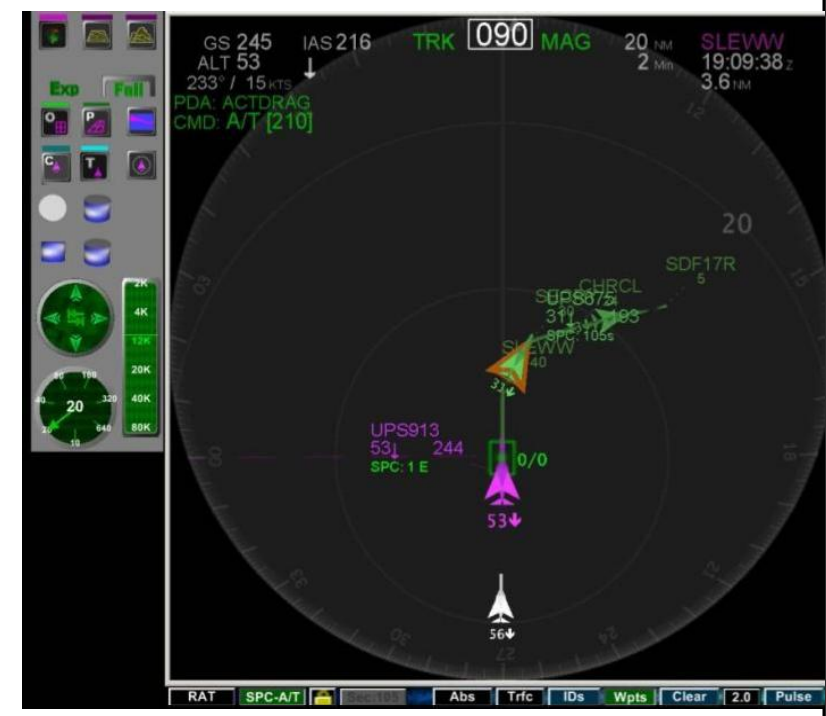

Figure 3. CSD interface display

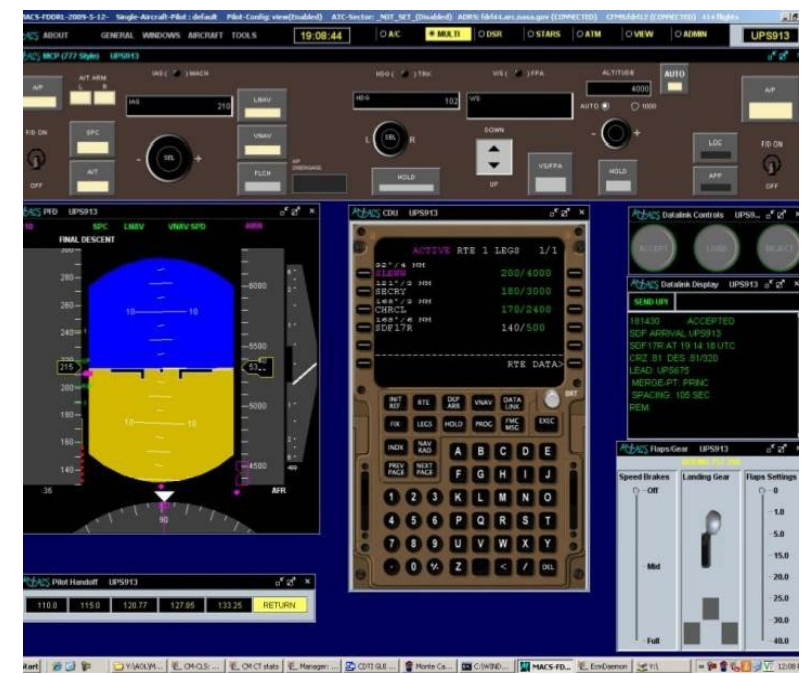

Figure 4. MACS interface Display

controllers of impending conflicts. When part of their roles and responsibilities, controllers resolved conflicts, and resequenced aircraft, with a trial planner tool that they could use to graphically generate a new proposed trajectory, and then datalink the selected route modifications to the pilot. The CSD (shown in 2D mode in Figure 3) provided pilots with a display of traffic and weather, plus CD\&R tools, flight path replanning tools, and interval management tools. The CSD provided an adjustable view of traffic, providing a $20-640 \mathrm{~nm}$ horizontal range, 2000 to 80000 feet vertical range, continuously adjustable 3D perspective views, and simulated airborne weather displays. The CSD could display all information in 2D (top down or profile) or in $3 \mathrm{D}$ views. The CSD also included an integrated trial planner, called the Route Assessment Tool 
(RAT). Similar to the controllers' trial planner, this tool allowed pilots to "grab" the current route and design new flight paths by stretching the route around weather. In Concepts 1 and 2 automated conflict alerting algorithms provided visual alerts when proposed routes created traffic conflicts. The RAT also provided feedback on how much delay the reroute generated. The CSD was integrated with the FMS allowing the pilot to execute the new route from the CSD. To alleviate controller workload, hand-offs and voice frequency changes were automated and check-ins were only required during merging and spacing operations. For controllers, IFR traffic was illuminated while TFR traffic was dimmed, unless in conflict with an IFR aircraft (in Concept 2).

\section{III.E. Distributed and Networked Simulation}

The simulation was distributed and networked over the internet with operators and/or participants located at different sites. Pilot stations and simulation management and other support stations were hosted in the FDDRL at the NASA Ames Research Center, while the two controllers and multiple ghost-controllers were located in the Center for Human Factors in Advanced Aeronautics Technologies (CHAAT) at California State University Long Beach (CSULB). Ghost-controllers controlled "ghost-sectors", which are airspace sectors adjacent to experimental sectors. Ghost sectors provide the airspace needed to facilitate the initiation and completion of hand-offs between sectors. Ghostcontroller stations were operated by students and faculty at CSULB's CHAAT. Multiple pseudo-pilots were located at the Systems Engineering Research Laboratory (SERL) at California State University Northridge (CSUN) and at the Human Integrated Systems Engineering Laboratory (HISEL) at Purdue University. Pseudo-pilot stations were operated by students and faculty at CSUN's SERL and Purdue University's HISEL. In addition, online situation awareness and workload probes administered at 3minute intervals asked both pilots and controllers to subjectively rate their workload and answer descriptive questions about their situation awareness. The readers are referred to [18-19, 21] for more details.

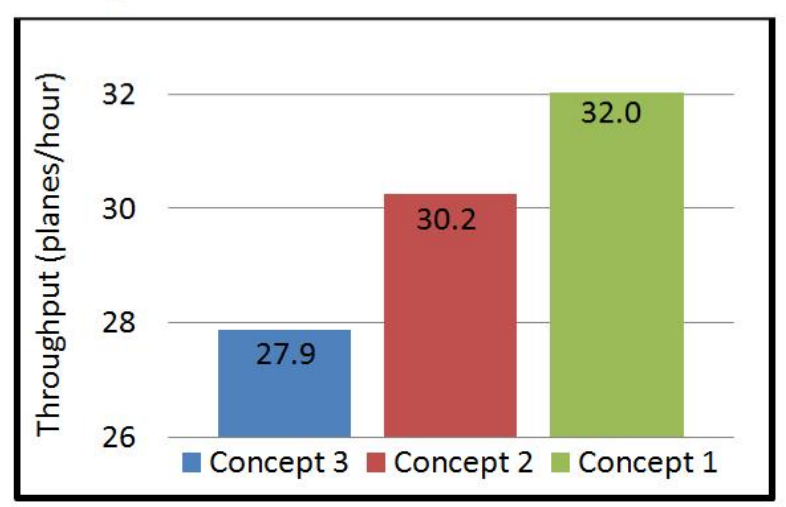

Figure 5. Throughput at CHRCL

\section{Results and Discussion}

Measures were submitted to a 3 (Concepts) x 2 (Display Type) repeated measures ANOVA via IBM SPSS 20.0. Greenhouse-Geisser adjustments were made for violations of sphericity when needed. The 3*IQR outlier test was used for all variables. Outliers were replaced with the means of the design cells in which they occurred. As noted above, due to data-collection equipment failure and malfunction occurred during the first week of the experiment, only data collected during the second week of this experiment is reported in this section.

\section{IV.A. System Performance}

To assess the feasibility of the function allocation concepts, the performance of the system was examined with five key metrics: 1) throughput at the final approach fix; 2) aircraft kinetic energy; 3) spacing interval variation at the merge point and at the final approach fix; 4) variation in speed and altitude along the descent; and 5) number of violation of speed and altitude constraints. These metrics provide the basis for examining the system's efficiency, stability, robustness to the en route weather perturbation, and compliance with safety constraints.

\section{Throughput}

The throughput (in planes per hour) for each concept was measured at the final approach fix, CHRCL, and shown in Figure 5. The difference in the throughput was significant $[\mathrm{F}(2,9)=5.764$, $\mathrm{p}<$ 0.05]. The throughput of Concept 1 was higher than those of Concepts 2 and 3, and is very close to the target throughput of 34.3 planes/hour (105 sec in trail spacing) designed into the experiment. 


\section{Aircraft kinetic energy}

The kinetic energy (KE) of the aircraft, shown in Figure 6, was calculated for the continuous descent approach (CDA) phase with the assumption that their masses are the same. In general, pilots were able to adhere to the target altitude profile but had trouble managing their speed. This translated into poor energy management as shown in Figure 6, where the energy is too low in Concept 2 and somewhat high in Concept 3 . Concept 3 was found to have significantly higher kinetic energy than Concepts 1 and 2 from CBSKT to CHRCL. In Concept 3, pilots were able to decrease the aircraft energy at CHRCL to the same level as in Concept 1. From SLEWW onward, Concept 2 was found to have the lowest kinetic energy.

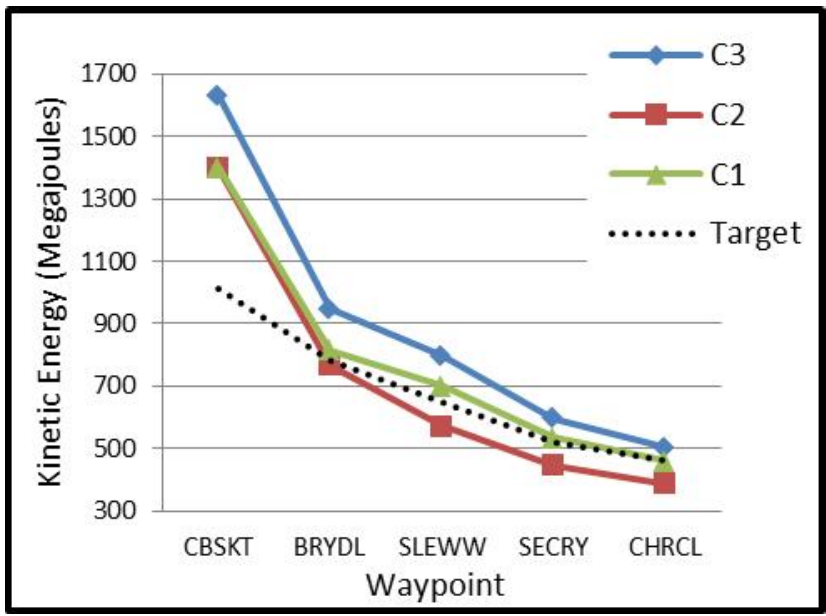

Figure 6. Mean descent KE across Concepts

The poor energy management can be further elucidated by examining mean $\mathrm{KE}$ target deviation shown in Figure 7. In all concepts, at the waypoint CBSKT, KE was severely off the target KE profile. This is especially significant for Concept 3, which had marginally higher deviation throughout the descent. Concept 2's deviation drops significantly after CBSKT and remains below target until CHRCL. Concept 1 maintained least deviation from the target KE profile, particularly at $\mathrm{CHRCL}(\triangle \mathrm{KE}=$ $0.23 \mathrm{MJ})$.

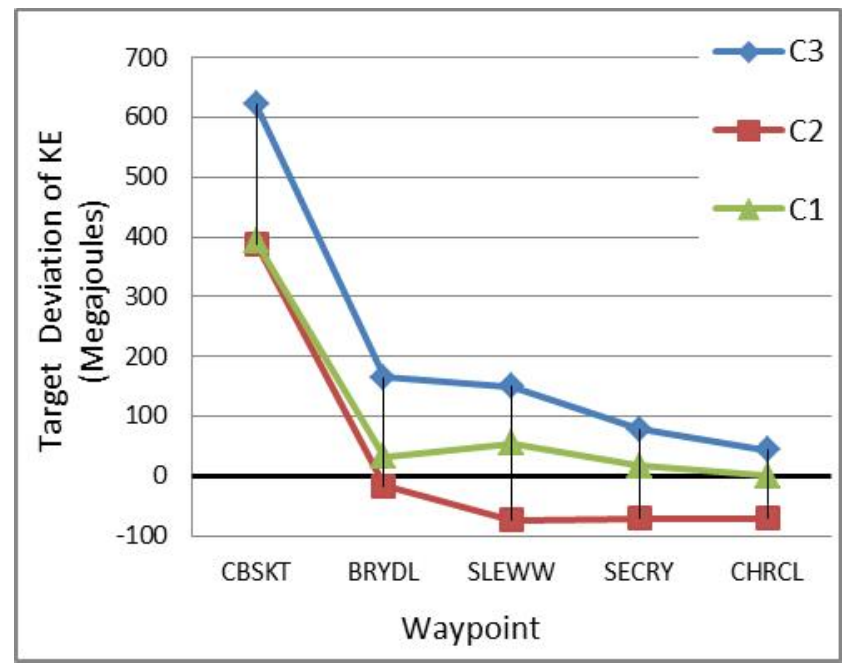

Figure 7. Mean KE deviation across Concepts

\section{Spacing}

Comparatively, the average spacing was significantly tighter in Concept 1 than in Concept 3 at all waypoints from CBSKT to CHRCL $(\mathrm{p}<.05)$, and closer to the target 105 seconds (See Figure 8). Mean spacing was significantly different between Concepts 2 and 3 only at CBSKT.

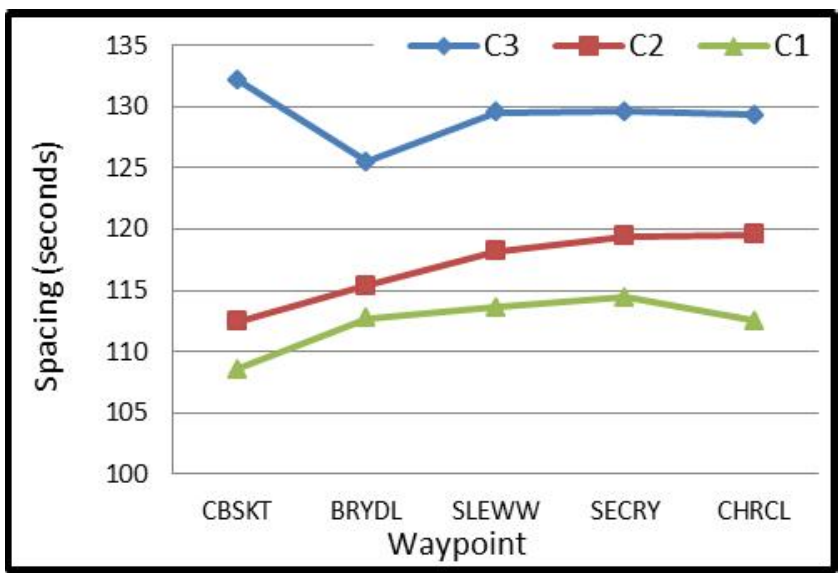

Figure 8. Mean Spacing across Concepts

\section{Altitude and Speed Profiles, and Constraint Violations}

For all waypoints along the descent, no significant differences in altitude were found across concepts. Altitude restriction violations were also calculated, but were relatively low for all concepts. Altitude target deviations did not exceed 80 feet for any waypoint in any concept. As illustrated in Figure 9, altitude across concepts was managed in a consistent and stable manner. 


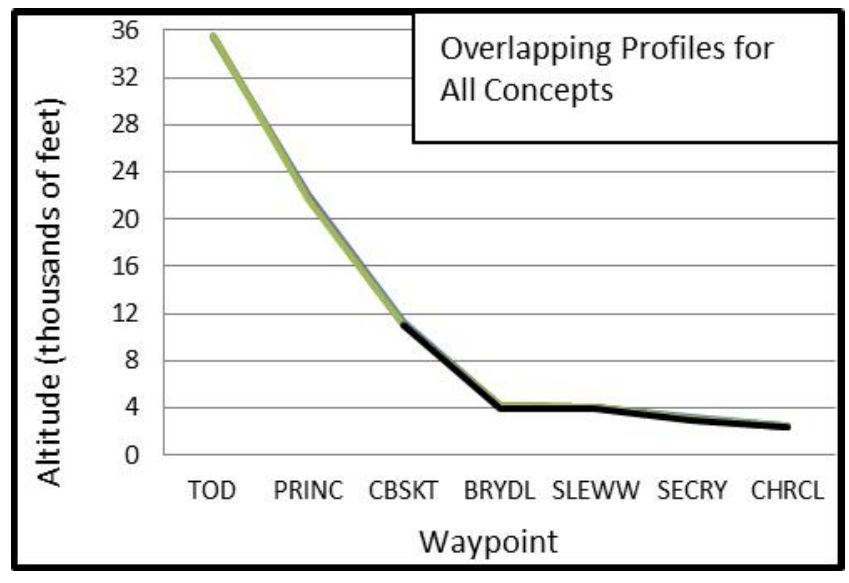

Figure 9. Altitude Profiles

The means of the indicated airspeed (IAS) of the three concepts are shown in Figure 10. Along the CDA path from CBSKT to CHRCL (see Figure 1), a speed restriction is defined as a deviation of \pm 10 knots from a waypoint's speed constraint (except at CHRCL where there is no lower limit). Table 3 shows the total violations at each waypoint with a breakdown of above and below deviations. Concept 2 had the highest number of violations, most of which were violations of the lower limit. Concept 3 had the second most violations, but these violations were mostly attributed to breaking the upper limit. These results agree with findings of higher mean IAS in Concept 3 and lower mean IAS in Concept 2, as shown in Figure 5. Analysis of IAS showed significant differences between Concepts 1 and 3 at $\operatorname{CBSKT}(\mathrm{F}(1.133,7.930)=11.219, \mathrm{p}<0.01)$. Speed was higher throughout the descent from CBSKT to CHRCL for Concept 3 than for Concepts 1 and 2 . Although aircraft were generally able to slow down and match the target profile by CHRCL, the consequence of carrying high energy did result in three upper limit speed violations at CHRCL in Concept 3, while both Concepts 1 and 2 had none. This highlights a critical shortcoming of Concept 3 in that it could not prevent more than $9 \%$ of the aircraft from violating an upper IAS limit at the final approach fix. Concept 2 matched Concept l's speed profile at CBSKT, but distinctly fell below Concept 1 at SLEWW $(\mathrm{F}(2,14)=54.005, \mathrm{p}<0.001)$. Concept 2 fell below the target profile, and had low speeds at CHRCL. Based on number of violations, Concept 1 's aircraft were able to match the target speed profile with better precision than the Concept 3 and Concept 2 aircraft.

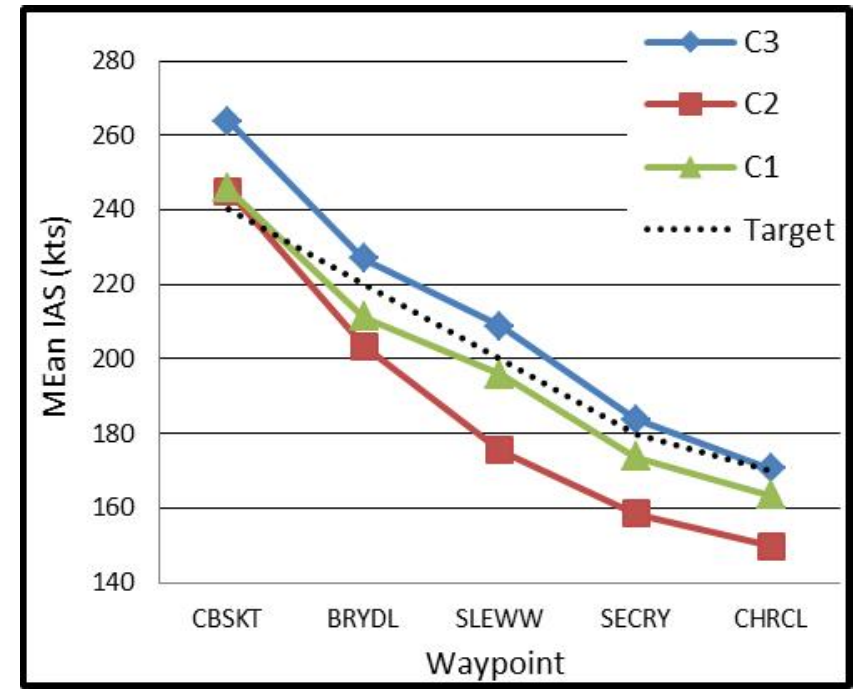

Figure 10. Mean descent IAS across Concepts

Table 3. Number of IAS Restriction Violations

\begin{tabular}{|l|l|l|l|}
\hline & Concept 1 & Concept 2 & Concept 3 \\
\hline Above & 18 & 15 & 56 \\
\hline Below & 37 & 62 & 4 \\
\hline Total & 55 & 77 & 60 \\
\hline
\end{tabular}

\section{IV.B. Situation Awareness and Workload}

The results on pilot and controller situation awareness and workload have been reported in a number of papers [18, 19, 21] and will only be summarized here. In general, it was found that pilots had the highest situation awareness in Concept 1 (Pilot Primary), when they were most actively engaged in and responsible for separation assurance. Conversely, pilots showed lowest situation awareness when they were least engaged in separation assurance in Concept 3 (Automation Primary). Workload was more dependent on the flight phase than function allocation concept, but trends in multiple workload metrics indicated that pilots had lowest workload when they are responsible for resolving traffic conflicts. This finding suggested that, for pilots, increases in workload were driven more by gaining situational awareness than by higher separation assurance responsibility. Controllers experienced highest workload in Concept 2 (Controller Primary) 
where they had the highest level of responsibility, and lowest workload in Concept 3 (Automation Primary). Overall, both pilots and controllers indicated in post-simulation questionnaires that all three concepts were workable and that they are comfortable with the concepts.

\section{IV.C. Discussion}

\section{Robustness to weather perturbation}

As shown in Figure 1, it was anticipated that the weather cells prior to the TOD and the merge point PRINC would disrupt the aircraft flow and create substantial variation in the initial conditions of the aircraft by the time they reach TOD or PRINC. However, previous analyses have shown that for all concepts there were no significant differences from the baseline profiles and no significant variation in terms of IAS, altitude, and KE at the TOD and the merge point PRINC [18]. That is, aircraft in each concept converged to a common flight state and had comparable initial conditions at the start of the CDA. The performance data from these previous examinations of this data (in terms of frequency with which aircraft entered and time spent in weather cells, time to pass weather and to reach TOD, the number of aircraft out of sequence or needing resequence, the number of spacing disengagement, and path stretch around weather cells) converged in showing that there was no significant differences in how pilots avoided the weather. The similar and comparable initial conditions at the TOD helped the aircraft tightly track the altitude profile without any statistically significant variations across all concepts as shown in Figure 9. Collectively these finding suggests that all concepts are robust to weather perturbation and performed comparably with one another.

\section{System Performance}

Based on the throughput and energy management and constraint violation results, pilot performance in Concept 1 (pilot primary) was superior to pilot performance in Concepts 2 (controller primary) and 3 (automation primary). However, it is interesting to note that upon beginning the descent, the pilots are no longer responsible for separation assurance because this responsibility was assigned to the controller in all concepts. In light of the findings from [18] mentioned above, that all concepts had comparable flight states at TOD and at the merge point, this suggests that the significant differences in throughput and energy management and violation of constraints are most likely due to the different degrees of pilot "in-the-loop" involvement in managing the spacing and the flight profile during the CDA. With Concept 1 , because pilots were assigned the separation responsibility before the CDA, they naturally would be actively involved in using the spacing tool to manage the spacing and in managing the descent even when they are no longer responsible for the separation. Consequently, they have the highest level of situation awareness among the concepts. With Concept 2, because the pilots had secondary responsibility in managing the separation before the CDA, they would be partially involved in managing spacing and the descent. Likewise, the controller was also not as actively involved in the loop because the spacing algorithm already handled the spacing between the aircraft and the controller only had to intervene when the spacing is projected to decrease below the minimum required separation. With concept 3 , because the pilots were not at all involved in the separation prior to the CDA, they would further fall "out-of-the-loop" after the CDA. This is evidenced in the lowest situation awareness that pilots had for this concept.

\section{Conclusions}

A key research issue of great interest to NextGen researchers and developers is the design of viable separation assurance concepts. While the results of this study are applicable to the conditions designed and manipulated in the experiment, they have a number of implications with respect to the design of the allocation of separation assurance responsibility between pilots, controllers, and automation. First, although the operation was stressed with severe weather disruption, all three concepts were robust to this perturbation in the presence of 3-time higher than current day traffic density. In combination, the system performance data and situation awareness and workload data, point to a general conclusion that all concepts are workable for the pilots and controllers. Thus, in the design of NextGen operational concepts, this study suggests that these three function allocation concepts are viable design alternatives that deserve further study or consideration for integration with other NextGen concepts such as Trajectory Operation or Trajectory Based Operation [1]. In the context of 
these concepts, it would be essential to further explore system performance under a number of newly proposed TO/TBO system architectural elements such as required time performance, open and closed trajectories, and dynamic windows (i.e., allowed flexibility in 4D trajectory). These new elements and concepts form a paradigm to facilitate conformance monitoring both on the flight deck and on the ground, and present new challenges in terms of designing operation procedures and display systems for conformance monitoring and alerting under TO/TBO.

Second, the results corroborate the fundamental automation design principle that the best form of automation design is one that involves the operator in the loop. This view is supported by this study, showing effects even though the required participation of the pilots was the same by the time they began the arrival phase. Because Concept 1(i.e., pilot primary) requires pilots to be actively engaged "in-the-loop" with separation assurance, it appears to motivate the pilots to continue to maintain this involvement even when they are no longer required to. In contrast, because Concepts 2 (controller primary) and Concept 3 (automation primary) take the pilots out-of-the-loop to a greater extent than Concept 1, the performance under these concepts are inferior to Concept 1.

\section{References}

[1] JPDO TBO Study Team, Trajectory-Based Operations (TBO) Operational Scenarios for 2025, Joint Planning and Development Office TBO Report Version 1.9.1, September 15, 2010.

[2] FAA, NextGen Mid-Term Concept of Operations for the National Airspace System, Initial Coordination Draft, Not Releasable, Version 1.0, June 30, 2009, Air Traffic Organization, NextGen \& Operations Planning, Research \& Technology Development, Air Traffic Systems Concept Development

[3] Prevot, T., Callantine, T., Lee, P., Mercer, J., Battiste, V., Johnson, W., Palmer, E., Smith, N., “ Cooperative air traffic management: A technology enabled concept for the Next Generation Air Transportation System," 5th USA/Europe Air Traffic management Research and Development Seminar, Baltimore, MD (2005).

[4] Prevot, T., Homola, J., Mercer, J., "Humanin-the-loop evaluation of ground-based automated separation assurance for NEXTGEN," 8th AIAA Aircraft Technology, Integration, and Operations Conference, Anchorage, Alaska (2008).

[5] SESAR Joint Undertaking, July 2010, ATM Master Plan Update Working Group Report. Edition $1.1 \quad$ www.atmmasterplan.eu/http://prismeoas.atmmasterplan.eu/atmmasterplan/faces/public/ur/ change_history.jspx.

[6] Duong, V., "FREER: Free-Route Experimental Encounter Resolution - Initial Results," EUROCONTROL Experimental Centre, EEC Paris Bretigny, October 1997.

[7] Erzberger, H., "The automated airspace concept," 4th USA/Europe Air Traffic Management R\&D Seminar, Santa Fe, NM (2001).

[8] Erzberger, H., "Transforming the NAS: The Next Generation Air Traffic Control System," 24th International Congress of the Aeronautical Sciences, Yokohama, Japan (2004).

[9] Granada, S., Dao, A.Q., Wong, D., Johnson, W.W., and Battiste, V, "Development And Integration Of A Human-Centered Volumetric Cockpit Display For Distributed Air-Ground Operations," Proc.12th International Symposium on Aviation Psychology, Oklahoma City, OK, 2005.

[10] Christopher D. Wickens, "A Model for Types and Levels of Human Interaction with Automation," Ieee Transactions On Systems, Man, And Cybernetics-Part A: Systems And Humans, Vol. 30, No. 3, May 2000.

[11] Barmore, B. E., Abott, T. S., Capron, W. R., and Baxley, B. T., "Simulation results for airborne precision spacing along continuous descent arrivals," 8th AIAA Aviation Technology, Integration, and Operations (ATIO) Conference, 1419 Sep. 2008, Anchorage, AK.

[12] Abbott, T. S., "Speed control law for precision terminal area," NASA Technical Memorandum 2002-211742. Hampton, VA: National Aeronautics and Space Administration. 
[13] Johnson, W.W., Battiste, V., Delzell, S., Holland, S., Belcher, S., Jordan, K., "Development and demonstration of a prototype free flight cockpit display of traffic information," Proceedings of the 1997 SAE/AIAA World Aviation Conference (1997).

[14] Williams, D., "Self-Separation in Terminal Areas Using CDTI," Proceedings of the Human Factors and Ergonomics Society Annual Meeting October 1983 vol. 27 no. 9 772-776.

[15] Federal Aviation Administration (2009). Advanced merging and spacing concept of operation for the nextgen mid-term. Unpublished manuscript.

[16] Grimaud, I., Hoffman, E., Pene, N., Rognin, L., and Zeghal, K., "Towards the Use of Spacing Instruction: Assessing the Impact of Spacing Tolerance on Flight Crew Activity, "Proceedings of the AIAA Guidance, Navigation, and Control Conference, Austin, TX, August 2003,

[17] Pritchett, A., Yankosky, L, "Simultaneous Design of Cockpit Display of Traffic Information and Air Traffic Management Procedures", SAE Transactions - Journal of Aerospace, 1998.

[18] Vu, K., Strybel, T., Battiste, V., Lachter, J., Dao, A., Summer, B., Ligda, S., Johnson, W., "Pilot Performance in Trajectory-Based Operations Under Concepts of Operation That Vary Separation Responsibility Across Pilots, Air Traffic Controllers, and Automation," International Journal of HumanComputer Interaction, 28:2, 107-118.

[19] Vu, K.-P. L., Strybel, T. Z., Kraut, J., Bacon, L. P., Minakata, K., Nguyen, J., Rotterman, A., Battiste, V. \& Johnson, W. " Pilot and Controller Workload and Situation Awareness Under Three Traffic Management Concepts," 29th Digital
Avionics Systems Conference, (pp. 4.A.5-1 -4.A.510) Salt Lake City, UT.

[20] NASA Ames Flight Deck Display Research Laboratory. http://humanfactors.arc.nasa.gov/ihh/cdti/cdti.html.

[21] Ligda, S., Dao, A., Vu, K., Strybel, T., Battiste, V., Johnson, W., "Impact of Conflict Avoidance Responsibility Allocation on Pilot Workload in a Distributed Air Traffic Management System," Proceedings of the Human Factors and Ergonomics Society 54th Annual Meeting, pp 55-59.

\section{Acknowledgements}

The simulation described in this paper was funded by NASA cooperative agreement NNA06CN30A, Metrics for Situation Awareness, Workload, and Performance in Separation Assurance Systems. The team developed and contributed to this simulation study includes the Flight Deck Display Research Laboratory at NASA Ames Research Center, the Center for Human Factors in Advanced Aeronautics Technologies at California State University Long Beach, the Systems Engineering Research Laboratory at California State University Northridge, and the Human Integrated Systems Engineering Laboratory at Purdue University.

\section{1st Digital Avionics Systems Conference} October 14-18, 2012 